\title{
NUMERICAL ANALYSIS OF THE EFFECT OF HYDRODYNAMICS AND OPERATING CONDITIONS ON BIODIESEL SYNTHESIS IN A ROTOR-STATOR SPINNING DISK REACTOR
}

\author{
Zhuqing Wen, Jerzy Petera* \\ Lodz University of Technology, Faculty of Process and Environmental Engineering, 90-924 Łódź, \\ Wólczańska 213, Poland
}

\begin{abstract}
A rotor-stator spinning disk reactor for intensified biodiesel synthesis is described and numerically simulated in the present research. The reactor consists of two flat disks, located coaxially and parallel to each other with a gap ranging from $0.1 \mathrm{~mm}$ to $0.2 \mathrm{~mm}$ between the disks. The upper disk is located on a rotating shaft while the lower disk is stationary. The feed liquids, triglycerides (TG) and methanol are injected into the reactor from centres of rotating disk and stationary disk, respectively. Fluid hydrodynamics in the reactor for synthesis of biodiesel from TG and methanol in the presence of a sodium hydroxide catalyst are simulated, using convection-diffusion-reaction multicomponent transport model with the CFD software ANSYSCFluent v. 13.0. Effect of operating conditions on TG conversion is particularly investigated. Simulation results indicate that there is occurrence of back flow close to the stator at the outlet zone. Small gap size and fast rotational speed generally help to intensify mixing among reagents, and consequently enhance TG conversion. However, increasing rotational speed of spinning disk leads to more backflow, which decreases TG conversion. Large flow rate of TG at inlet is not recommended as well because of the short mean residence time of reactants inside the reactor.
\end{abstract}

Keywords: biodiesel synthesis, ANSYSCFluent, rotor-stator reactor, backflow, operating conditions

\section{INTRODUCTION}

The widely used method for biodiesel production is transesterification of oil or fat feedstock with methyl alcohol under alkaline conditions in a liquid-liquid environment. Transesterification is a liquidliquid two phase reaction. Reaction rate can be limited by mass transfer between the oil and alcohol phases. Therefore, the efficiency of mass transfer is of importance for improving production capacity, reducing process cost and equipment size. Many intensification technologies have been explored to enhance contact and contact area between the two liquid phases and decrease resistance to mass transfer in the reactor. SDR (short for spinning disk reactor), which includes spinning disks (also known as rotors) and stationary disks (also known as stators), is one of the process intensification technologies employing high gravity-centrifugal force fields caused by the rotation of rotors. Extensive studies using this technology have shown that process time, reactant inventories, and impurity levels can be reduced by up to 99\% (Brechtelsbaueret al., 2001; Cafieroet al., 2002; Clifford et al., 2009).

The hydrodynamics inside the rotor-stator gap are mostly important to quantify the performance (such as conversion of reactants, mass transfer rate) of the SDR. Researchers have investigated the fluid flow inside rotor-stator gap with and without superposed through flow. The boundary layers of fluid flow inside rotor-stator gap without superposed through flow are supposed to be a combination of Von 
Kármán layer along the rotating disk (Von Kármán, 1921) and Bodewadt layer along the stationary disk (Bodewadt, 1940).The Von Kármán layer is characterized by a net centrifugal radial velocity and a net negative axial (towards the disk) fluid velocity; analogously, the Bodewadt layer has a net centripetal velocity and a net positive axial (away from the disk) velocity. The Von Kármán layer and Bodewadt layer form the basis for depicting the flow structures inside the rotor-stator gap.

Investigations indicate that either a Von Kármán layer develops on the rotor alone, or Von Kármán layer and Bodewadt layer develop on both the rotor and the stator depending on the boundary conditions of the disk (Brady and Durlofsky, 1987; Lopez, 1998). In the former case, the bulk fluid is at rest and only a Von Kármán layer is formed along the spinning disk (Stewartson, 1953). In the latter case, the exact flow structure is mainly determined by the rotational Reynolds number (also known as channel Reynolds number) and the gap ratio of the system (Batchelor, 1951; Daily and Nece, 1960).

The present research simulated the fluid flow inside SDR gap with feedstocks injected from inlets. Introducing an externally applied through flow into SDR gap alters the flow structures depicted above. Studies show that in the case of a superposed centrifugal through flow, at low radial positions, the tangential velocity (swirl velocity) decreases to zero outside the Von Kármán layer, and the radial velocity is positive (radially outward) for all axial positions (Haddadi and Poncet, 2008; Phadke and Owen, 1988; Poncet et al., 2005a; Poncet et al., 2005b; Soo, 1958). At higher radial positions, the flow structure depends on the gap ratio employed (Haddadi and Poncet, 2008; Poncet et al., 2005b), the radial velocity is centrifugal along the spinning disk and centripetal along the stationary disk.

An engineering model of the fluid flow patterns inside the inter-disk space of rotor-stator was proposed, previously depicted by De Beer et al. (2014). The flow through a rotor-stator cavity can be described as a combination of radial plug flow and ideally mixed region. The radius where flow pattern changes from plug flow to ideally mixed flow decreases with increasing rotational Reynolds number and gap ratio, and increases with increasing superposed through flow rate. In the present research, the SDR developed to explore the possibility of improving the efficiency of biodiesel production is described and used, as shown in Fig.1. ANSYSCFluent 13.0 is chosen to simulate and analyse fluid hydrodynamics in this reactor for the synthesis of biodiesel using convection-diffusion-reaction multicomponent transport model. Simulation results show similarity to the study carried out by De Beer et al. (2014), and details are described below. There is an obvious occurrence of back flow near the stator from the outlet. The size of the backflow zone increases with the rotational speed increase; it decreases with the gap size reduction and with the flowrate increase. It is also demonstrated that backflow is undesirable in the present study by analysing the reactant conversion in SDR.

\section{EXPERIMENTAL SETUP AND MECHANISM OF ALKALI-CATALYSED TRANSESTERIFICATION REACTION}

The experimental setup was built and described by Qiu and Weatherley (Qiu et al., 2012), who are our collaborative partners from Department of Chemical and Petroleum Engineering, Kansas University, USA. We use their experiment data to verify our simulation results. The SDR system consists of two parallel coaxial disks: one stationary and the other rotational with controllable rotational speed. The disks in the present research are separated by an adjustable gap. The two immiscible liquid feed stocks, oil and alcohol, are pumped axially into the reactor from the centres of the spinning disk and the stationary disk, respectively. The model reaction system chosen for study is methyl-esterification of canola oil in the presence of a sodium hydroxide catalyst to form biodiesel and glycerol. Before being pumped into SDR, sodium methoxide is prepared by dissolving sodium hydroxide in methanol. The experimental setup is shown in Fig. 1. 


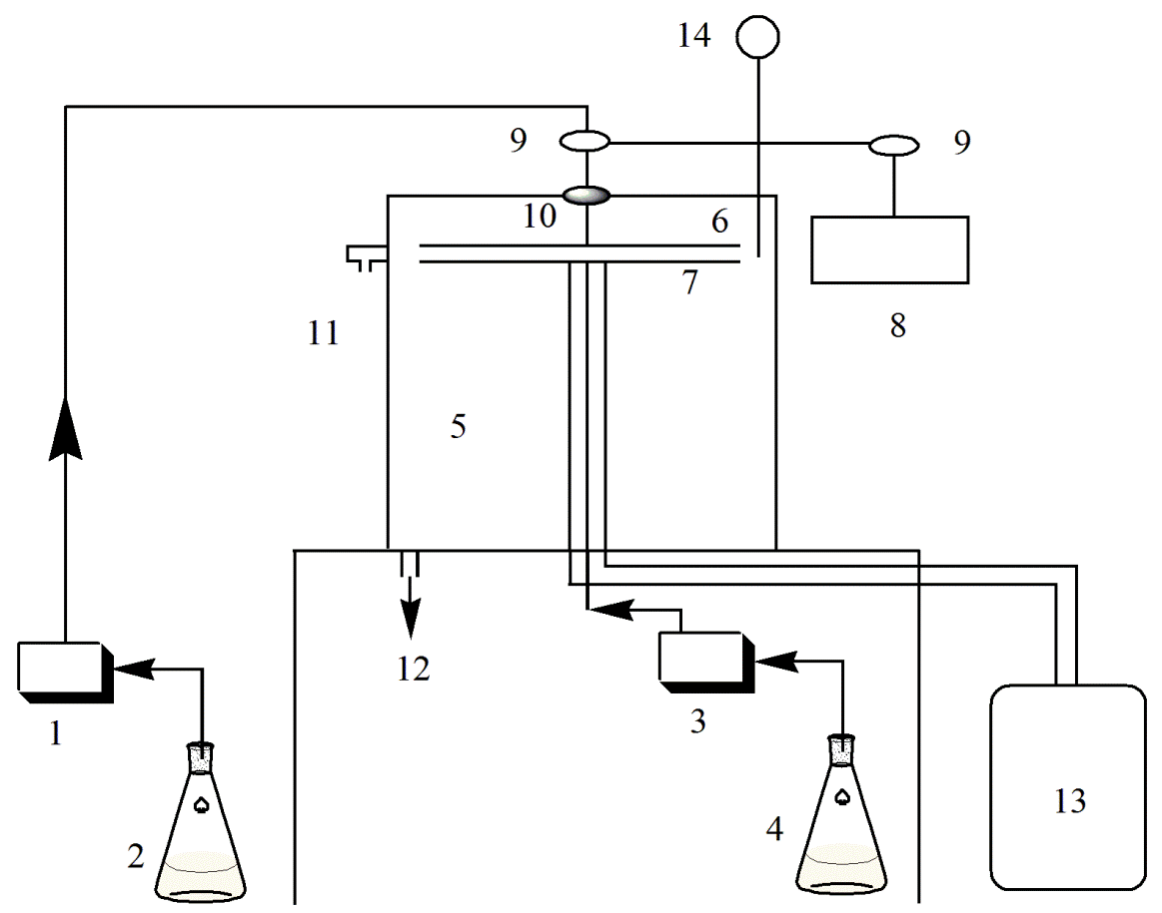

Fig. 1. Experimental setup of the intensive SDR for biodiesel synthesis;

1 - peristaltic pump; 2 - canola oil vessel; 3 - digital piston pump; 4 - sodium methoxide vessel; 5 - cylinder; 6 - rotating disk; 7 - stationary disk; 8 - variable-speed DC motor; 9 - pulley; 10 - bearing; 11 - sampling point;

12 - products drainage; 13 - heating circulator; 14 - thermometer

As mentioned above, biodiesel is mainly made by transesterification of vegetable oils and animal fats with alcohol in the presence of catalysts. Vegetable oils and animal fats typically consist of triglycerides (TG) which are esters of free fatty acids with the trihydric alcohol, glycerol.

During the transesterification of TG, there are three stepwise and reversible reactions with intermediate formation of diglycerides (DG) and monoglycerides (MG) resulting in the production of methyl esters $\left(\mathrm{RCOOCH}_{3}\right.$, biodiesel) and glycerol (GL) as shown in chemical equations I-III (Freedmanet al., 1986; Noureddini and Zhu, 1997). The alcohol to TG molar ratio, catalyst type, reaction time and reaction temperature can affect the transesterification at different levels (Freedmanet al., 1984).

$$
\begin{aligned}
& \mathrm{TG}+\mathrm{CH}_{3} \mathrm{OH} \underset{k_{2}}{\stackrel{k_{1}}{\longrightarrow}} \mathrm{DG}+\mathrm{RCOOCH}_{3} \\
& \mathrm{DG}+\mathrm{CH}_{3} \mathrm{OH} \underset{k_{4}}{\stackrel{k_{3}}{\longrightarrow}} \mathrm{MG}+\mathrm{RCOOCH}_{3} \\
& \mathrm{MG}+\mathrm{CH}_{3} \mathrm{OH} \underset{k_{6}}{\stackrel{k_{5}}{\longrightarrow}} \mathrm{GL}+\mathrm{RCOOCH}_{3}
\end{aligned}
$$

In order to describe the chemical kinetics of the transesterification reaction, the following equations are used.

$$
\begin{aligned}
& -r_{I}=k_{1} \cdot C_{\mathrm{TG}} \cdot C_{\mathrm{CH}_{3} \mathrm{OH}^{-}} k_{2} \cdot C_{\mathrm{DG}} \cdot C_{\mathrm{RCOOCH} 3} \\
& -r_{I I}=k_{3} \cdot C_{\mathrm{DG}} \cdot C_{\mathrm{CH}_{3} \mathrm{OH}^{-}} k_{4} \cdot C_{\mathrm{MG}} \cdot C_{\mathrm{RCOOCH} 3} \\
& -r_{I I I}=k_{5} \cdot C_{\mathrm{MG}} \cdot C_{\mathrm{CH}_{3} \mathrm{OH}^{-}} k_{6} \cdot C_{\mathrm{GL}} \cdot C_{\mathrm{RCOOCH} 3}
\end{aligned}
$$

where $k_{i}(i=1, \ldots, 6)$ represents the respective kinetic constant that follows the Arrhenius equation:

$$
k_{i}=A_{i} \mathrm{e}^{-E_{i} /(R T)} \quad i=1,2, \ldots, 6
$$




\section{SIMULATION METHODOLOGY}

\subsection{Mesh information and boundary conditions}

The CFD software, ANSYSCFluent v.13.0 was chosen to simulate and analyse the hydrodynamics performance of SDR. The effects of gap size between spinning disk and stationary disk, rotational speed of spinning disk, and TG flow rate at inlet are investigated.

It is important to appropriately mesh the computational domain to get accurate simulation results. To obtain satisfactory mesh, it is necessary to simplify the real construction of SDR. As can be seen from Fig. 1, the region of interest in the experimental setup is the space between spinning disk and stationary disk. Besides, this construction is axisymmetric, hence, only half of it is simulated. The magnification of the reaction part and the computational domain are shown in Figs. 2 and 3.

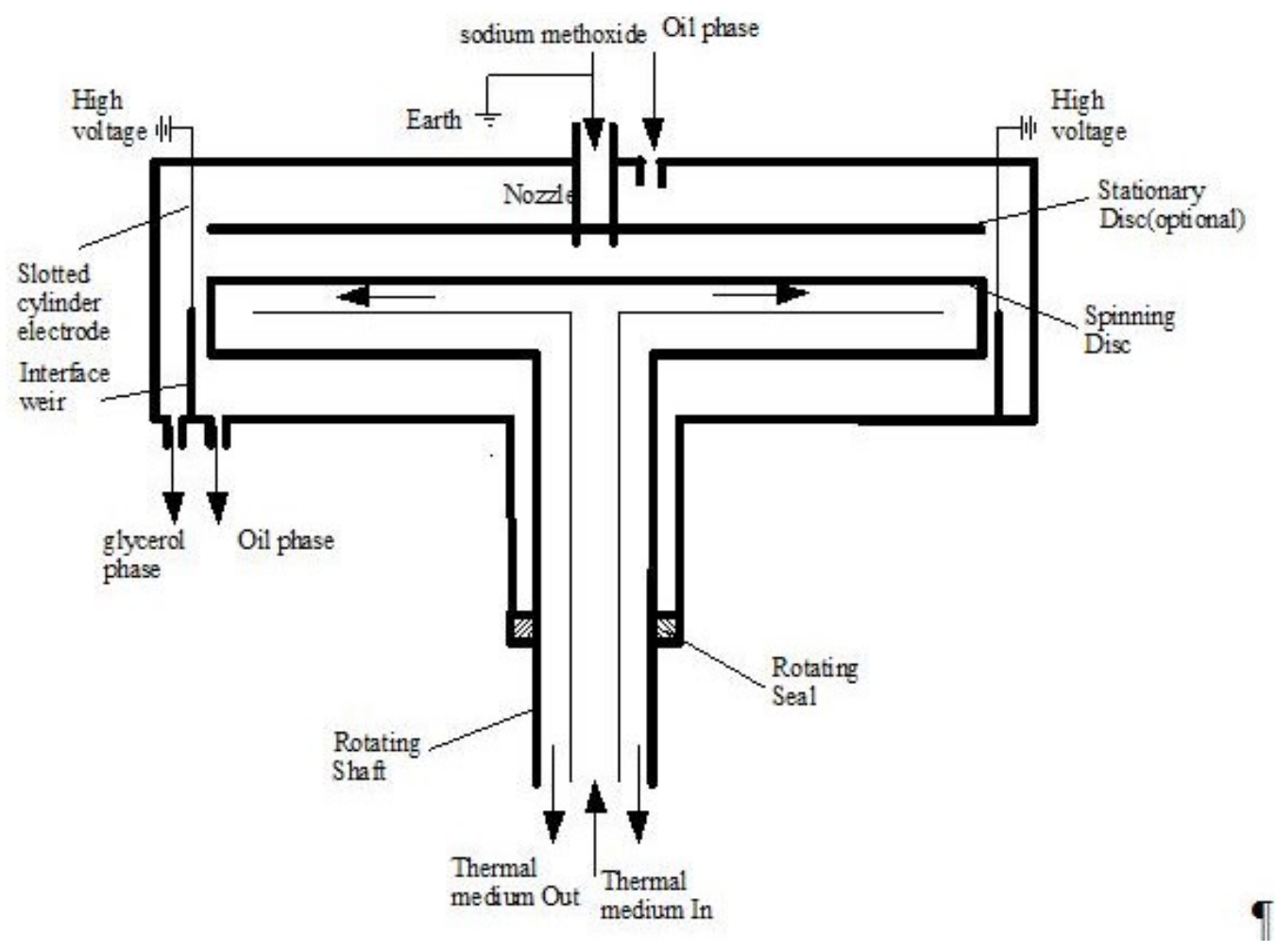

Fig. 2. Magnification of the reaction part

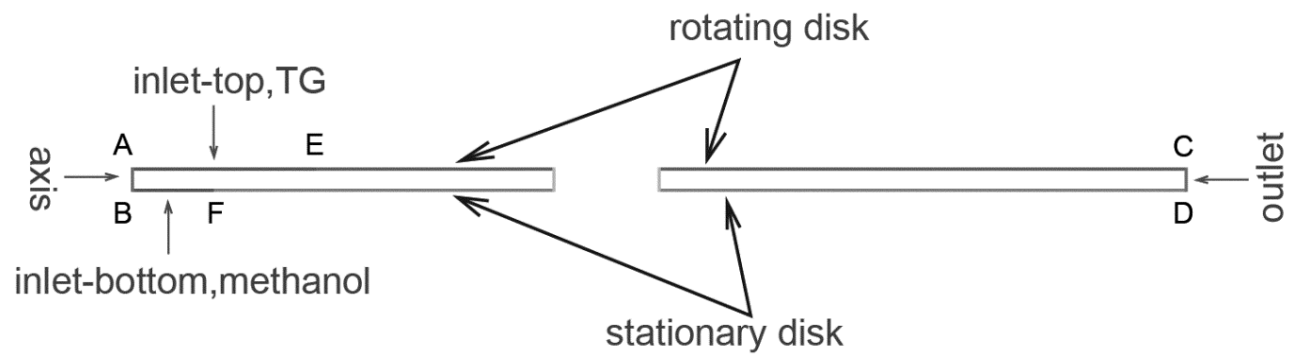

Fig. 3. Simplified computational domain of SDR

Dimensions of the domain, based on the SDR construction and after simplification, are as follows. Domain radius AC: $50 \mathrm{~mm}$. Gap size between rotor and stator is adjustable, applied gap size AB: $0.1 \mathrm{~mm}, 0.15 \mathrm{~mm}, 0.2 \mathrm{~mm}$. Radius of inlet-top AE and inlet-bottom BF are $1.75 \mathrm{~mm}$ and $0.77 \mathrm{~mm}$. As 
introduced before, TG and sodium methoxide (methanol in the simulation) are pumped into the reactor through the centres of spinning disk and stationary disk, respectively. When the volume flow rate of TG and methanol at inlet-top and inlet-bottom are $5.77 \times 10^{-8} \mathrm{~m}^{3} \mathrm{~s}^{-1}(3.84 \mathrm{ml} / \mathrm{min})$ and $1.3 \times 10^{-8} \mathrm{~m}^{3} \mathrm{~s}^{-1}$, the injecting velocities of TG and methanol are $0.006 \mathrm{~ms}^{-1}$ and $0.007 \mathrm{~ms}^{-1}$; when the volume flow rate of TG and methanol at the inlet-top and the inlet-bottom are $1.12 \times 10^{-7} \mathrm{~m}^{3} \mathrm{~s}^{-1}(6.69 \mathrm{ml} / \mathrm{min})$ and $2.9 \times 10^{-8} \mathrm{~m}^{3} \mathrm{~s}^{-1}$, the injecting velocities of TG and methanol are $0.0116 \mathrm{~ms}^{-1}$ and $0.0156 \mathrm{~ms}^{-1}$, respectively. The boundary conditions will be introduced in detail below.

The computational domains are meshed with ANSYS meshing tool in Workbench 13. The mesh information for different gap size is shown in Table 1. Three different gap sizes between rotor and stator are investigated in the present simulation. Six cases are simulated for each gap size, differing from the rotational speed of spinning disk. Moreover, two flow rates of TG at inlet are studied when gap size is $0.2 \mathrm{~mm}$. The overall boundary conditions are summarised in Tables 2 and 3 .

Table 1. Mesh information for different gap sizes

\begin{tabular}{|c|c|c|c|}
\hline Gap size, $\mathrm{mm}$ & Cells & Faces & Nodes \\
\hline 0.1 & 50000 & 105010 & 55011 \\
\hline 0.15 & 94444 & 194460 & 100017 \\
\hline 0.2 & 100035 & 205088 & 105054 \\
\hline
\end{tabular}

Table 2. Overall boundary conditions in the simulation

\begin{tabular}{|l|c|c|c|c|c|c|}
\cline { 2 - 7 } \multicolumn{1}{c|}{} & $\mathrm{AB}$ & $\mathrm{AE}$ & $\mathrm{BF}$ & $\mathrm{EC}$ & $\mathrm{FD}$ & $\mathrm{CD}$ \\
\hline Boundary type & $\mathrm{Axis}$ & $\begin{array}{c}\text { Velocity- } \\
\text { inlet }\end{array}$ & $\begin{array}{c}\text { Velocity- } \\
\text { inlet }\end{array}$ & Wall & Wall & Outflow \\
\hline Length, mm & $\begin{array}{c}0.1,0.15, \\
0.2\end{array}$ & 1.75 & 0.77 & 48.25 & 49.23 & $\begin{array}{c}0.1,0.15, \\
0.2\end{array}$ \\
\hline Temperature, K & - & 298.15 & 298.15 & 298.15 & 298.15 & - \\
\hline Velocity, ms ${ }^{-1}$ & - & $\begin{array}{c}0.006 \text { or } \\
0.0116\end{array}$ & $\begin{array}{c}0.007 \text { or } \\
0.0156\end{array}$ & - & - & - \\
\hline $\begin{array}{l}\text { Rotational speed of } \\
\text { spinning disk, rpm }\end{array}$ & - & - & - & $\begin{array}{c}1000- \\
2000\end{array}$ & - & - \\
\hline
\end{tabular}

Table 3. Rotational speed of spinning disk for each case

\begin{tabular}{|l|c|c|c|c|c|c|}
\cline { 2 - 6 } \multicolumn{1}{c|}{} & case 1 & case 2 & case 3 & case 4 & case 5 & case 6 \\
\hline $\begin{array}{l}\text { Rotational speed of } \\
\text { spinning disk, rpm }\end{array}$ & 1000 & 1200 & 1400 & 1600 & 1800 & 2000 \\
\hline
\end{tabular}

\subsection{Equations solved in the simulation}

The computational domain is simplified to a $2 \mathrm{D}$ axisymmetric geometry as described above, and the external body forces are not considered in the present research, hence conservation equations solved in the simulation are shown as follows (ANSYSCFluent v.13.0 Theory Guide, 2010).

The continuity equation is given as

$$
\frac{\partial \rho}{\partial t}+\frac{\partial}{\partial x}\left(\rho v_{x}\right)+\frac{\partial}{\partial r}\left(\rho v_{r}\right)=0
$$


The axial momentum conservation equation is given by

$$
\frac{\partial \rho}{\partial t}\left(\rho v_{x}\right)+\frac{1}{r} \frac{\partial}{\partial x}\left(r \rho v_{x} v_{x}\right)+\frac{1}{r} \frac{\partial}{\partial r}\left(r \rho v_{r} v_{x}\right)=-\frac{\partial p}{\partial x}+\frac{1}{r} \frac{\partial}{\partial x}\left[r \mu 2 \frac{\partial v_{x}}{\partial x}-\frac{2}{3}(\nabla \cdot v)\right]+\frac{1}{r} \frac{\partial}{\partial r}\left[r \mu\left(\frac{\partial v_{x}}{\partial r}+\frac{\partial v_{r}}{\partial x}\right)\right] \text { (4) }
$$

where

$$
\nabla \cdot v=\frac{\partial v_{x}}{\partial x}+\frac{\partial v_{r}}{\partial r}+\frac{v_{r}}{r}
$$

The radial momentum conservation equation is given by

$$
\begin{gathered}
\frac{\partial}{\partial t}\left(\rho v_{r}\right)+\frac{1}{r} \frac{\partial}{\partial x}\left(r \rho v_{x} v_{r}\right)+\frac{1}{r} \frac{\partial}{\partial r}\left(r \rho v_{r} v_{r}\right)=-\frac{\partial p}{\partial r}+\frac{1}{r} \frac{\partial}{\partial x}\left[r \mu\left(\frac{\partial v_{x}}{\partial r}+\frac{\partial v_{r}}{\partial x}\right)\right]+\frac{1}{r} \frac{\partial}{\partial r}\left[r \mu 2 \frac{\partial v_{r}}{\partial r}-\frac{2}{3}(\nabla \cdot v)\right]+ \\
-2 \mu \frac{v_{r}}{r^{2}}+\frac{2}{3} \frac{\mu}{r}(\nabla \cdot v)+\rho \frac{v_{w}^{2}}{r} \\
\frac{\partial}{\partial t}\left(\rho v_{w}\right)+\frac{1}{r} \frac{\partial}{\partial x}\left(r \rho v_{x} v_{w}\right)+\frac{1}{r} \frac{\partial}{\partial r}\left(r \rho v_{r} v_{w}\right)=\frac{1}{r} \frac{\partial}{\partial x}\left[r \mu \frac{\partial v_{w}}{\partial x}\right]+\frac{1}{r^{2}} \frac{\partial}{\partial r}\left[r^{3} \mu \frac{\partial}{\partial r}\left(\frac{v_{w}}{r}\right)\right]-\rho \frac{v_{r} v_{w}}{r}
\end{gathered}
$$

There is no additional source for species in the present study. ANSYS@Fluent predicts the local mass fractions of each species through the solution of convection-diffusion-reaction equation for each species. The general form of this equation is shown as follows (ANSYS@Fluent v.13.0 Theory Guide, 2010):

$$
\frac{\partial}{\partial t}\left(\rho Y_{i}\right)+\nabla \cdot\left(\rho v Y_{i}\right)=-\nabla \cdot J_{i}+R_{i}
$$

The channel Reynolds number (also known as rotational Reynolds number) inside SDR cavity in the present research can be calculated using equation given by Sinnott (2005) and modified here to the following form

$$
\operatorname{Re}=\frac{\rho N D d}{\mu}
$$

All the channel Reynolds numbers used in the present research imply that the flow in this SDR is laminar flow. This can be demonstrated by the previous studies (Daily and Nece, 1960; Djaouiet al., 2001; de Beer et al., 2014). Hence, the diffusion flux of species $i, J_{i}$, which arises due to concentration gradients, can be written as

$$
J_{i}=-\rho D_{m, i} \nabla Y_{i}
$$

As generally known, the two feed stocks TG and methanol are immiscible and they form a two-phase liquid-liquid system after being injected into the SDR, thus the reactions are limited by mass transfer among reagents. The gap sizes between the spinning disk and the stationary disk which are used in the current SDR vary in the range of $0.1 \mathrm{~mm}$ to $0.2 \mathrm{~mm}$. As a result, a high velocity gradient is present between the spinning disk and the stationary disk, creating a high shear force occurring in the gap. Fast rotation of spinning disk amplifies further the effect of shearing in the gap thus reducing the mass transfer resistance by shortening the diffusive micro-distance transport.

We apply the homogeneous model describing the reacting mixture as a Newtonian suspension of droplets with viscosity depending on local volumetric fraction of dispersed phase. We want to obtain a qualitative but possibly rigorous explanation of experimentally observed increase of the conversion at higher shear inside the gap. We think that including "ad hoc" the explicit two-phase model facilities 
(like velocity field for each phase, droplet break up, etc.) could obscure the main phenomena in our case. Similar conclusions were drawn by Rudniak et al. (2004).

We assume that droplets do not disappear during the whole process, in agreement with Qiu (2010) who mentioned that the reaction mixture passes from a biphasic (methanol and oil) system to a biphasic (methyl ester-rich and glycerol-rich) system during transesterification. The reaction generally preserves volume and in the dispersed phase the methanol substrate is at least partially replaced by glycerol product. Zhou and Boocock (2006) investigated phase distribution of methanol, glycerol, and catalyst in the transesterification of soybean oil. Investigation revealed that $42.0 \%$ of the alcohol, $2.3 \%$ of the glycerol, and only $5.9 \%$ of the catalyst were present in the ester-rich phase at steady state starting with an initial condition of $6: 1$ alcohol/oil molar ratio and catalyst concentration (1.0 wt\% sodium methoxide). The reaction conditions used in Zhou and Boocock's investigation are similar to those utilized in the present research, and the investigation further demonstrated the existence of the droplet mainly consisting of methanol, glycerol and catalyst during the whole transesterification process. Thus we propose that the enhanced conversion at higher rotational speeds is caused by faster mass transport in the interface diffusional layer, the thickness of which decreases at higher shear in the gap between the disks. The diameter distribution is also expected to tend towards smaller droplets, which at approximately the same total volume of dispersed phase means a development of the interface area. Figure 4 below illustrates the process.
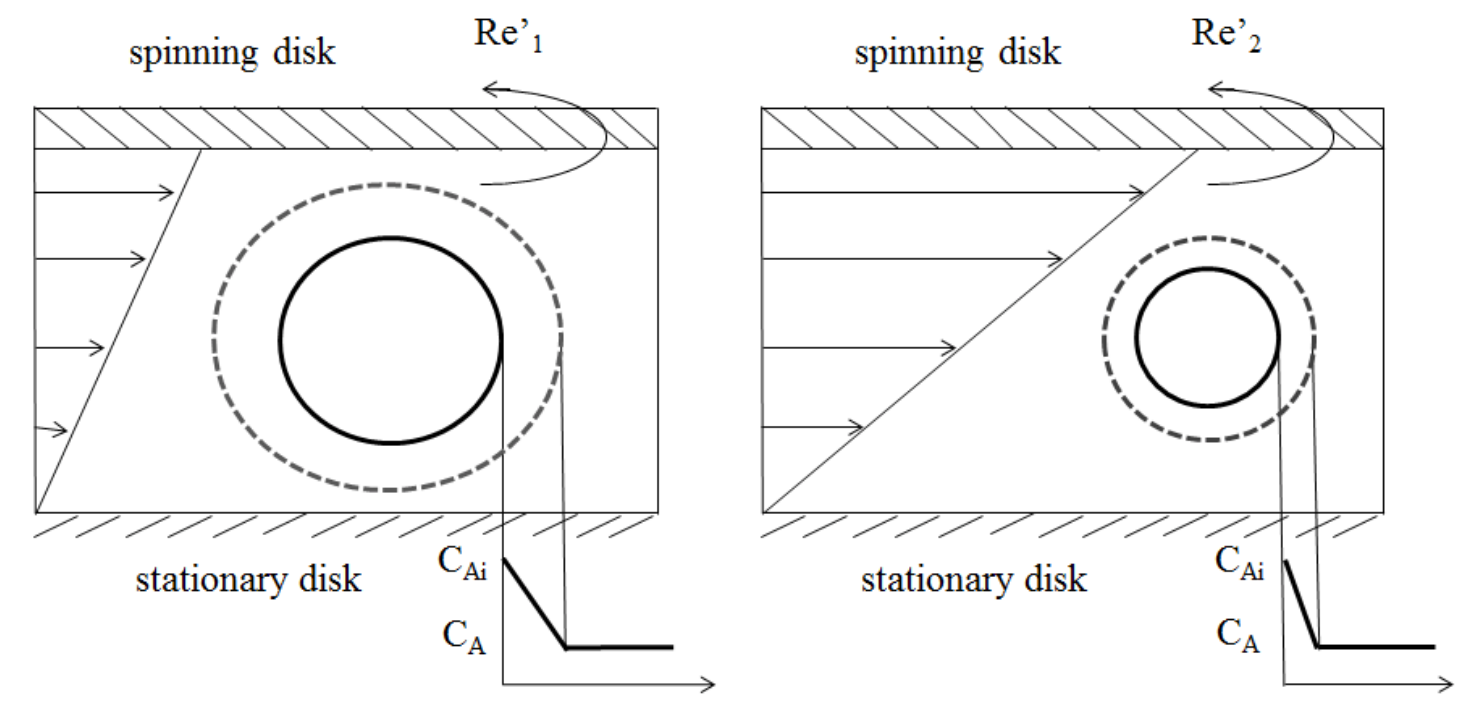

Fig. 4. Illustration of the effect of shear force on effective diffusivity; $C_{A i}$ - concentration of product at the interface, $C_{A}$ - concentration of product in the continuous phase

In the model approach this is equivalent to an increase of the mixture effective diffusivities $D_{m, i}$ controlled by the gap size and rotational speed. In the present research we propose the following formula for modification of the mixture diffusivities to account for the shearing influence, which means effective diffusivity:

$$
D_{m, i}=D_{m, i o}\left\lfloor 1+A\left(R e^{\prime}\right)^{a}\right\rfloor
$$

where $D_{m, i o}$ are understood as pure diffusivities for individual components.

The value of $A$ defines the magnitude of $D_{m}$ under different operating conditions and is set as 0.022 . The value of a is deduced from the exponent on Reynolds number in empirical correlations among Sherwood number, Reynolds number and Schmidt number, which describe mass transfer in the continuous phase around drops. Sandoval-Robles et al. (1980) have shown how the exponent on Reynolds number changes with the value of Reynolds number from $1 / 3$ for Stokes regime to $1 / 2$ for 
boundary-layer theory, and to $2 / 3$ for a surface renewal mechanism or turbulent conditions (Richardson, 1963).

The particle diffusion Reynolds number, $R e$ ', is modified from the particle Reynolds number proposed by Rusconi and Stone (2008).

$$
R e^{\prime}=\frac{\rho N D d}{\mu}\left(\frac{D}{d}\right)^{2}
$$

For the calculation of chemical reactions, the laminar finite-rate model is used. The net source of chemical species $i$ due to reaction $R_{i}$ is computed as the sum of the Arrhenius reaction sources over the $N_{R}$ reactions that the species participate in:

$$
R_{i}=M_{w, i} \sum_{r=1}^{N_{R}} R_{i, r}^{\prime \prime}
$$

The molar rate of creation/destruction of species $i$ in reaction $r\left(R_{i, r}^{\prime}\right)$ in Eq. (13)) is given by

$$
R_{i, r}^{\prime}=\left(\vartheta_{i, r}^{\prime \prime}-\vartheta_{i, r}^{\prime}\right)\left(k_{f, r} \prod_{j=1}^{N_{R}}\left[C_{j, r}\right]^{\eta^{\prime}{ }_{j, r}}-k_{b, r} \prod_{j=1}^{N_{R}}\left[C_{j, r}\right]^{\eta^{\prime \prime} j, r}\right)
$$

The forward rate constant for reaction $r, k_{f, r}$, is computed using the Arrhenius expression.

$$
k_{f, r}=A_{r} e^{-E_{r} / R T}
$$

In this research, the kinetic rate constant and activation energy data are obtained and modified on the basis of data in Table 4.

Table 4. Chemical kinetics data for all reactions (Noureddini and Zhu, 1997)

\begin{tabular}{|c|c|c|c|}
\hline Parameter & $\begin{array}{c}\text { Reaction I } \\
(T G \rightarrow D G)\end{array}$ & $\begin{array}{c}\text { Reaction II } \\
(D G \rightarrow M G)\end{array}$ & $\begin{array}{c}\text { Reaction III } \\
(M G \rightarrow G L)\end{array}$ \\
\hline Kinetic rate constant, $k_{i}$ & $8.333 \times 10^{-4}$ & $3.583 \times 10^{-3}$ & $4.033 \times 10^{-3}$ \\
\hline Activation energy, $E_{i}$ & $5.5 \times 10^{7}$ & $8.309 \times 10^{7}$ & $2.687 \times 10^{7}$ \\
\hline
\end{tabular}

For reversible reaction the backward rate constant $k_{b, r}$ for reaction $r$ is determined by following expression:

$$
k_{b, r}=\frac{k_{f, r}}{K_{r}}
$$

where $K_{r}$ is the equilibrium constant for the reaction $r$, determined by the expression:

$$
\begin{gathered}
K_{r}=\exp \left(\frac{\Delta S_{r}^{0}}{R}-\frac{\Delta H_{r}^{0}}{R T}\right) \cdot\left(\frac{P_{a t m}}{R T}\right)^{\sum_{i=1}^{N}\left(\vartheta^{\prime \prime}{ }_{i, r}-\vartheta^{\prime}{ }_{i, r}\right)} \\
\frac{\Delta S_{r}^{0}}{R}=\sum_{i=1}^{N}\left(\vartheta^{\prime \prime}{ }_{i, r}-\vartheta^{\prime}{ }_{i, r}\right) \frac{S_{i}^{0}}{R} \\
\frac{\Delta H_{r}^{0}}{R T}=\sum_{i=1}^{N}\left(\vartheta^{\prime \prime}{ }_{i, r}-\vartheta^{\prime}{ }_{i, r}\right) \frac{H_{i}^{0}}{R T}
\end{gathered}
$$




\subsection{Modelling assumptions}

For the purpose of modelling and in order to simplify the calculation, initial assumptions are made as follows:

- Due to the fact that canola oil, as a mixture of fatty acids, consists mainly of oleic acid, triolein $\left(\mathrm{C}_{57} \mathrm{H}_{104} \mathrm{O}_{6}\right)$ is chosen as a representation of TG, accordingly, diolein $\left(\mathrm{C}_{39} \mathrm{H}_{72} \mathrm{O}_{5}\right)$ is chosen as a representation of DG, monoolein $\left(\mathrm{C}_{21} \mathrm{H}_{40} \mathrm{O}_{4}\right)$ is chosen as a representation of MG, and methyl oleate $\left(\mathrm{C}_{19} \mathrm{H}_{36} \mathrm{O}_{2}\right)$ is chosen as a representation of the resulting methyl ester (biodiesel).

- Feed stocks from inlet-top and inlet-bottom are TG and methanol, respectively. Molar ratio of methanol to TG is $6: 1$, and concentration of $\mathrm{NaOH}$ is $1.0 \mathrm{wt} \%$. There is no explicit occurrence of a catalyst; although the presence of the catalyst is taken into account by modification of the rate constants in the kinetic equations.

- The problem is nearly isothermal because the thermal boundary conditions assume perfect thermal control, thus all physicochemical properties are nearly constant. In the present research, the reaction temperature is chosen as $25^{\circ} \mathrm{C}$ for all cases.

- At the start the whole domain (the gap) is filled with TG at rest and from this point the process starts by injecting substrates TG and methanol.

- The order of magnitude for diffusion coefficients for vegetable oils is $10^{-9} \mathrm{~m}^{2} \mathrm{~s}^{-1}$ (Cussler, 1997). Egbuna et al. (2013) investigated diffusivities for the diffusion of palm oil into different solvents, the values ranged from 6.47 to $7 \times 10^{-9} \mathrm{~m}^{2} \mathrm{~s}^{-1}$. Based on this, a pure diffusivity value for each component in the reacting mixture, Dm,i0, is assumed to be $7 \times 10^{-9} \mathrm{~m}^{2} \mathrm{~s}^{-1}$ in the present research.

- The feedstock for inlet-top is canola oil (ConAgra Foods, USA) in experiments (Qiu, 2010). Generally the content of free fatty acids in canola oil is $0.4-1.2 \%$, which can result in saponification reaction (Przybylski, 2007). Owing to the low level of free fatty acids content in canola oil and relatively small amount of catalyst used in the experiments, saponification side reaction is ignored in the present research.

\section{SIMULATION RESULTS}

\subsection{Simulation results of hydrodynamics inside SDR gap}

Figure 5(a) shows the flow field in the SDR gap, Fig. 5(b) is the magnification of the flow field near the inlet, as marked in square in Fig. 5(a), Fig. 5(c) is the magnification of the flow field near the outlet, as marked in oval in Fig. 5(a). In the present research, the liquids injected into SDR flow outwards at low radial positions as shown in Fig. 5(b), while at high radial positions, there are two situations. The liquid accelerates near the rotor outwards because of centrifugal force caused by the spinning disk rotation, while a backflow near the stator is created as a result of the continuity conservation, which prevents the liquid from breakage as shown in Fig. 5 (c), the inwards flow is identified as backflow in the present study.

The simulation of the flow pattern inside the SDR gap is consistent with the research conducted by De Beer et al. (2014). They proposed and depicted an engineering model of the fluid flow patterns inside the inter-disk space of rotor-stator. The flow through a rotor-stator cavity can be described as a combination of radial plug flow and ideally mixed region, the radial liquid velocity profile between the rotor and the stator which is dominated by plug flow has a parabolic shape over the height between the rotor and the stator. In the mixed region, the radial velocity profile of the liquids dominated by boundary layer formation. The centrifugal Von Kármán boundary layer is present at the rotor, and the centripetal Bodewadt boundary layer is present at the stator. 


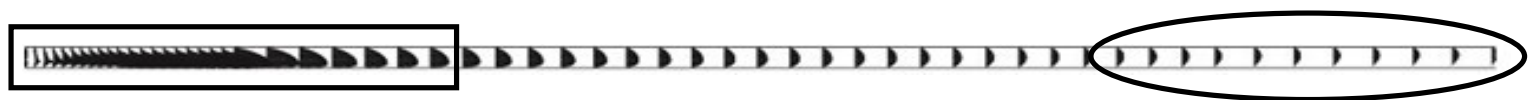

a

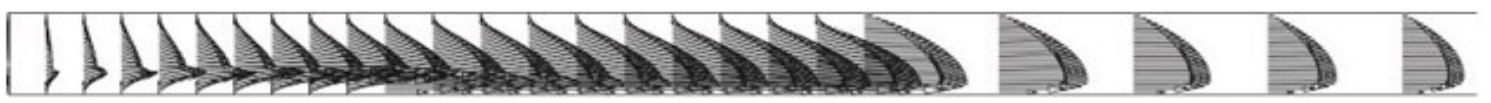

b

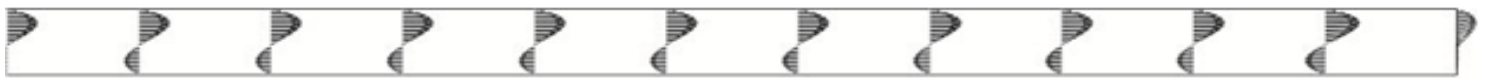

c

Fig. 5. The flow field in the SDR gap (a), the inlet (b) and the outlet (c)

Figure 6 presents the backflow zone inside SDR gap showing a characteristic pattern. The vertical position of the picture is justified by the ANSYSCFluent technicality. When the axisymmetric geometry is modelled, the direction of swirl axis is assumed to be the $\mathrm{X}$ axis and the computational domain is situated above the $\mathrm{X}$ axis. A pattern such as the one marked in purple indicates where the backflow meets with the predominant outward stream, the mixed liquids flow together outwards outlet after meeting. The boarder of this backflow zone may move with varying rotational speed of spinning disk, gap size between rotor and stator, and flow rate of TG at the inlet.

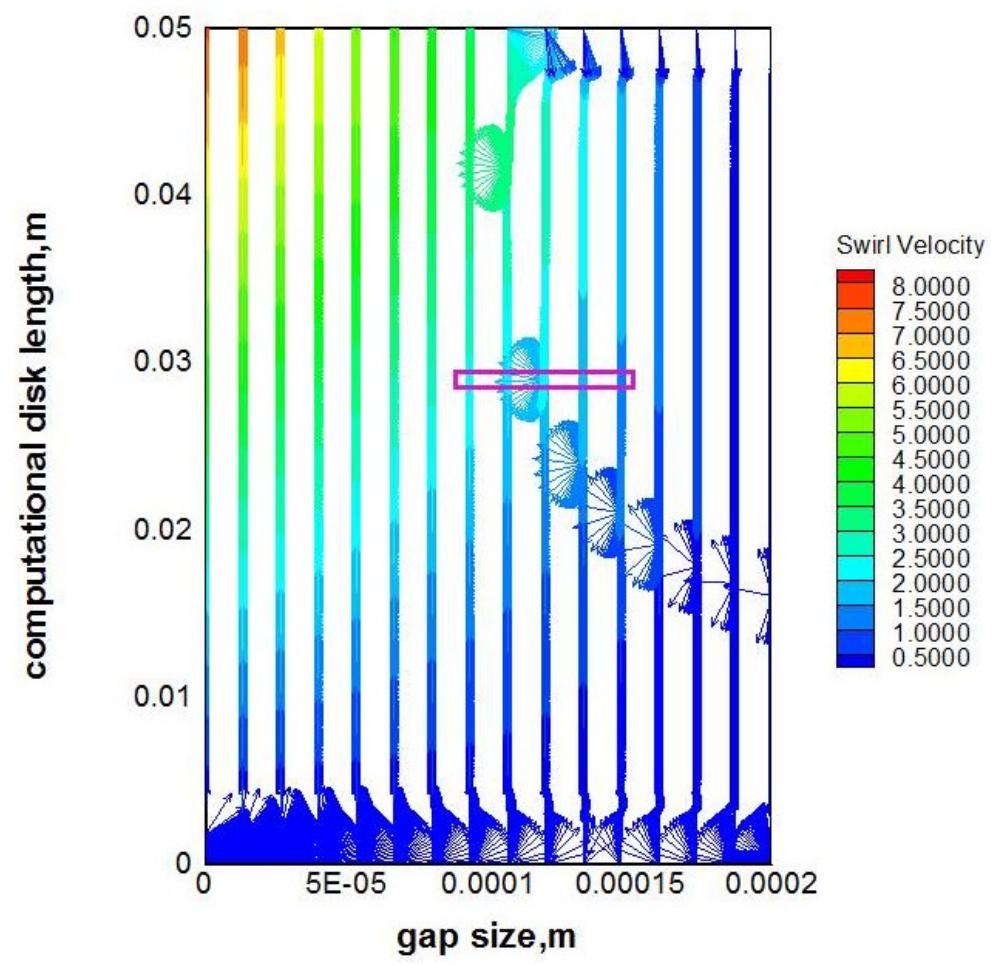

Fig. 6. Overall backflow zone within SDR gap in form of velocity vectors (The geometry proportions are intentionally changed.)

To investigate how operating conditions affect the size of backflow zone, three gap sizes of SDR are simulated. For each gap size, six rotational speeds of spinning disk are studied. For the gap size $0.2 \mathrm{~mm}$, two flow rates of TG at inlet are simulated. The sizes of backflow zones under different operating conditions are shown in Figs. 7 and 8.

Figure 7 demonstrates that the size of backflow zone increases as the rotational speed of spinning disk increases, and decreases with decreasing gap size between rotor and stator. In other words, faster 
rotational speed of spinning disk and bigger gap size between spinning disk and stationary disk lead to more backflow in SDR gap. On the other hand, as is evident in Fig. 8, the size of backflow zone decreases with increasing flow rate of TG at the inlet. It indicates that larger flow rate of TG at inlet leads to less backflow in SDR gap.

The simulation results reflected in Figs. 7 and 8 are also consistent with the research carried out by De Beer et al. (2014). The authors concluded that the radius where flow pattern changes from plug flow to ideally mixed flow decreases with increasing rotational Reynolds number and gap ratio, and increases with increasing superposed through flow rate.

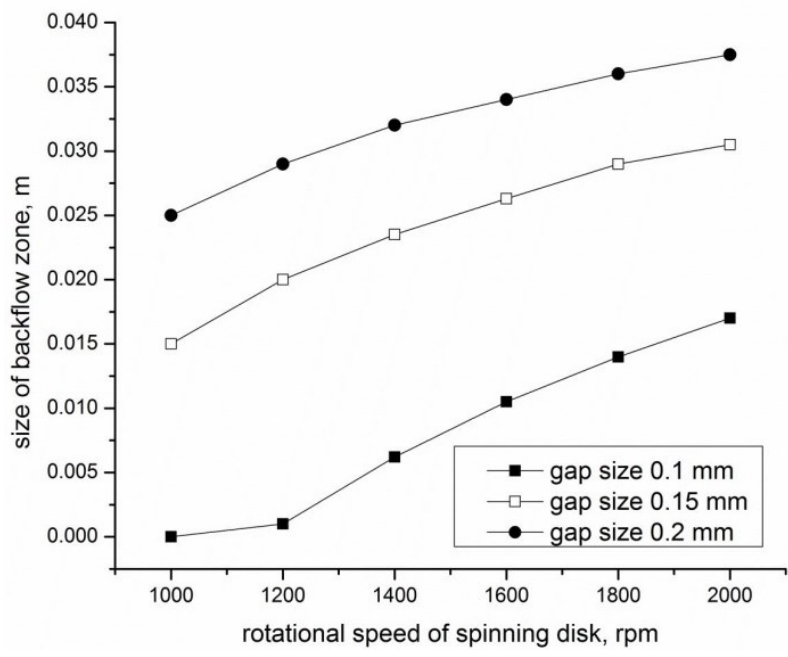

Fig. 7.Changes of backflow zone size with varying rotational speed and gap size

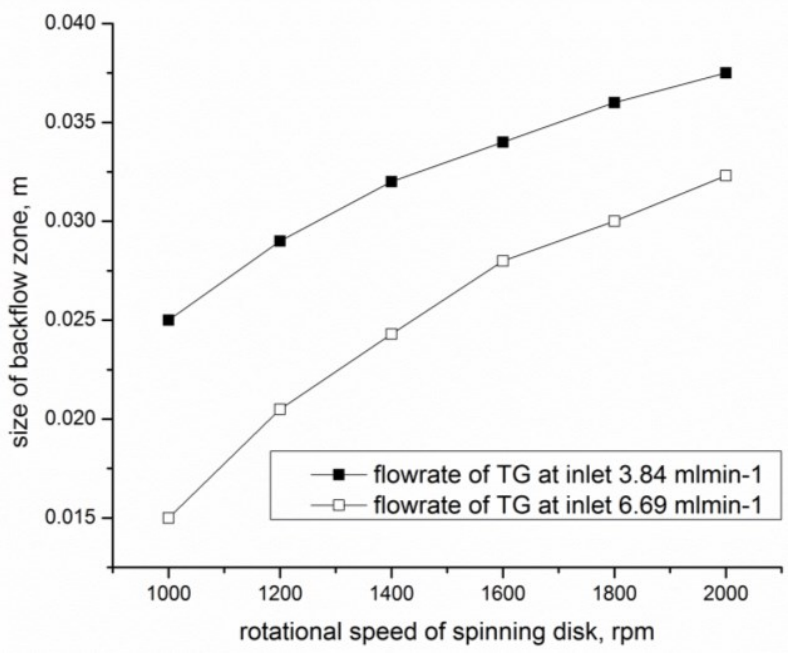

Fig. 8. Changes of backflow zone size with varying flow rate of TG at inlet

\subsection{Analysis of effect of operating conditions on TG conversion in SDR}

SDR, identified as having small gap size and fast rotational speed, is an efficient intensification technology for liquid-liquid two-phase reaction. The small gap sizes induce a high shear force to act on the reactants in the gap, leading to better mass transfer. Fast rotational speed of spinning disk is also of importance to intensify mixing and helps to eliminate the mass transfer resistance in the SDR gap. Since the rate of TG transesterification is limited by mass transfer between oil and alcohol phases due to their immiscibility, small gap size and fast rotational speed apparently result in higher TG conversion.

To determine values of TG conversion $\kappa$, the following equation is used:

$$
\kappa=\frac{C_{0} \cdot Q_{v 0}-C_{k} \cdot Q_{v k}}{C_{0} \cdot Q_{v 0}} \cdot 100 \%
$$

All of the above variables $C_{0}, C_{k}, Q_{v 0}, Q_{v k}$, can be discerned with ANSYSCFluent Reports.

The verification experiments conducted by Qiu and Weatherley using the experimental setup are shown in Fig. 1. The Feed stocks are TG and methanol, molar ratio of methanol to TG is $6: 1$, and concentration of catalyst $\mathrm{NaOH}$ is $1.0 \mathrm{wt} \%$. The experiment conditions are the same as those used in the simulation, as shown in Table 5. Simulation results and experimental data of TG conversion varying with different gap size and rotational speed are displayed in Figs. 9, 10 and 11. 
Table 5. Verification experiment conditions

\begin{tabular}{|c|c|c|c|c|}
\cline { 2 - 5 } \multicolumn{1}{c|}{} & Temperature, $\mathrm{K}$ & Gap size, $\mathrm{mm}$ & $\begin{array}{c}\text { Flow rate of TG, } \\
\mathrm{ml} / \mathrm{min}\end{array}$ & $\begin{array}{c}\text { Rotational speed of } \\
\text { spinning disk, rpm }\end{array}$ \\
\hline Value & 298.15 & $0.1 / 0.15 / 0.2$ & $3.84 / 6.69$ & $\begin{array}{c}1000 / 1200 / 1400 / \\
1600 / 1800 / 2000\end{array}$ \\
\hline
\end{tabular}

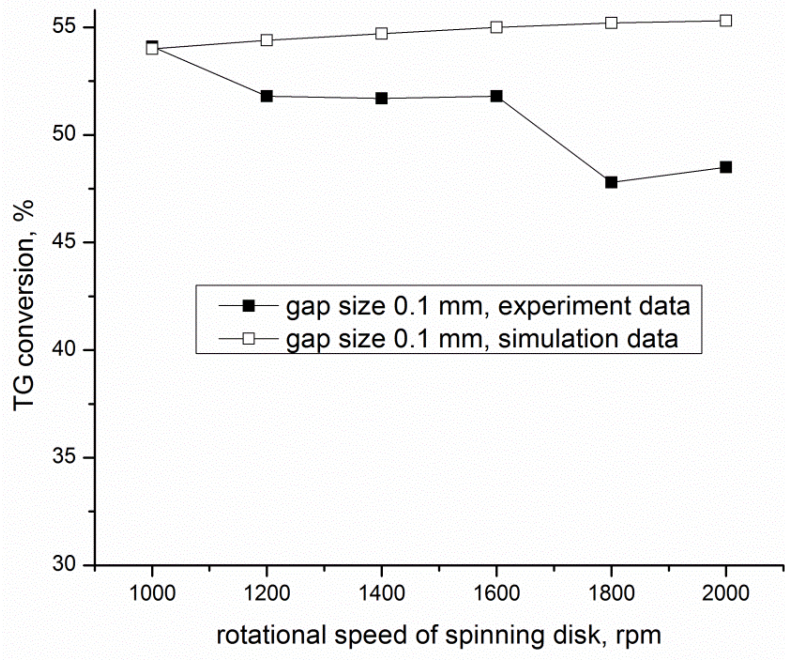

Fig. 9. Effect of rotational speed of spinning disk on TG conversion when gap size is $0.1 \mathrm{~mm}$

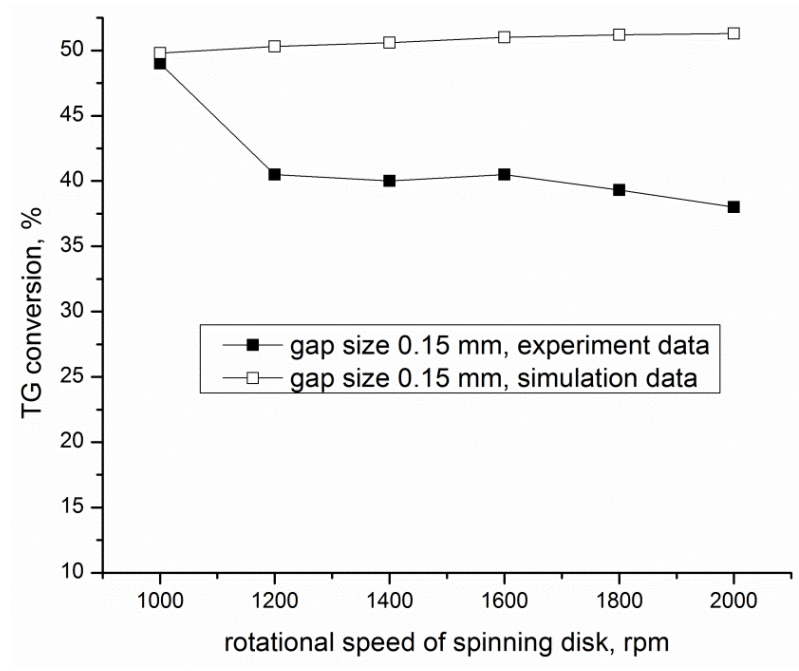

Fig. 10. Effect of rotational speed of spinning disk on TG conversion when gap size is $0.15 \mathrm{~mm}$

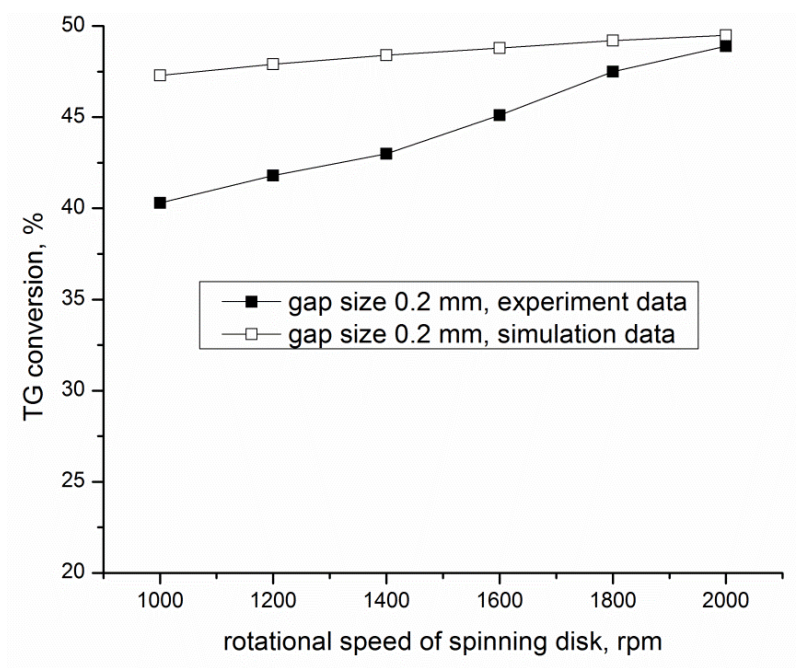

Fig. 11. Effect of rotational speed of spinning disk on TG conversion when gap size is $0.2 \mathrm{~mm}$

From Fig.9, Fig.10 and Fig.11 we can state that TG conversion increases as gap size decreases, simulation results show good agreement with experiment data. The simulation results indicate that TG conversion increases slightly with increasing rotational speed. While the experimental data show that when gap sizes are $0.1 \mathrm{~mm}$ and $0.15 \mathrm{~mm}$, TG conversion decreases as rotational speed of spinning disk increases. Fig. 7 reveals that faster rotational speed of spinning disk and bigger gap size between spinning disk and stationary disk lead to more backflow in SDR gap, we conclude that TG conversion decreases as size of backflow zone increases, i.e., backflow is undesirable in biodiesel synthesis in SDR. 
We give below a rigorous original explanation of the observed negative effect of increasing backflow zone on the conversion (which is enhanced either with increasing gap size or increasing rotational speed).

First, let us recall that the overall chemical equation for transesterification of TG and methanol producing biodiesel and glycerol can be written as

$$
\mathrm{TG}+3 \mathrm{CH}_{3} \mathrm{OH} \stackrel{\text { catalyst }}{\longleftrightarrow} 3 \mathrm{RCOOCH}_{3}+\mathrm{GL}
$$

The reaction rate for the overall chemical equation of the transesterification, $r_{t}$, can be calculated by Eq. (21)

$$
r_{t}=k_{f} \cdot C_{T G} \cdot C_{\text {methanol }}^{3}-k_{b} \cdot C_{R C O O C H 3}^{3} \cdot C_{G L}
$$

Although it is a reversible reaction, the forward reaction is dominant under the present operating conditions thus the overall reaction rate depends mainly on concentrations of TG and methanol.

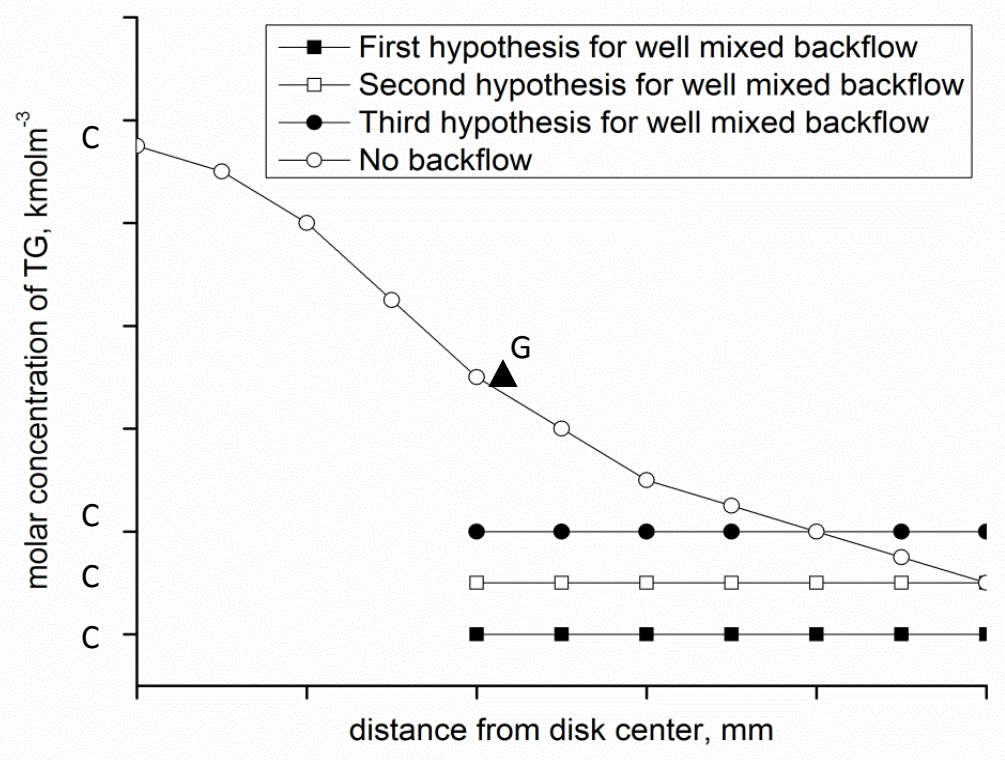

Fig. 12. Molar concentration of TG changes along SDR gap under different hypothesis

The reactants TG and methanol are injected into the SDR from centres of rotor and stator respectively, and without any backflow the concentration of TG will decrease along the SDR gap. This is illustrated in Fig. 12 by the hollow circles connecting points of concentrations $C_{1}$ to $C_{3}$.

Naturally, back mixing results in a tendency to level off all the concentrations in the backflow zone, including TG concentration. Suppose a back mixing starts at a distance marked by the point $G$ in Fig. 12, which implies a constant concentration of TG as illustrated by one of the three horizontal lines in this figure.

Suppose now hypothetically that TG concentration in the back-mixing zone is equal or less than $C_{3}$ in Fig. 12. This would mean that the conversion is better than in case of no back-mixing described by the hollow circles. On the other hand, however, according to Eq. (21), rate of reaction would be smaller in the back-mixing zone which obviously contradicts the initial hypothesis as the final lower substrate concentration, such as $C_{4}$ cannot be achieved with lower reaction rate. Subsequently the final substrate concentration must be bigger than $C_{3}$, such as $C_{2}$ in Fig. 12 which means that the conversion is worse in case when the back-mixing occurs. 
There is an exception when the gap size is equal to $0.2 \mathrm{~mm}$, for which TG conversion increases with the rotational speed increase, even though size of the backflow zone increases. Probably the larger gap size produces smaller shear force and the reaction rate of transesterification is still limited by the mass transfer between the two phases. In this situation growing rotational speed enhances the interface of mixing between reactants and consequently the TG conversion increases. The effect of flow rate of TG at inlet is also investigated in the present research. Fig. 8 shows that size of backflow zone is smaller when bigger flow rate of TG at inlet $(6.69 \mathrm{ml} / \mathrm{min})$ is utilized. Theoretically, TG conversion is supposed to be higher when flow rate of TG at inlet is bigger than that obtained when smaller flow rate of TG at inlet $(3.84 \mathrm{ml} / \mathrm{min})$ is used. However, both simulation results and experimental data show that TG conversion is smaller when bigger flow rate of TG at inlet is used, as shown in Fig.13. This can be explained by the fact that the mean residence time of the reactants in SDR when flow rate of TG at inlet is $6.69 \mathrm{ml} / \mathrm{min}$ is much shorter than that when flow rate of TG at inlet is $3.84 \mathrm{ml} / \mathrm{min}$. The mean residence time is too short for the reactants to react when flow rate is $6.69 \mathrm{ml} / \mathrm{min}$, that is why bigger flow rate of TG at inlet leads to smaller TG conversion.

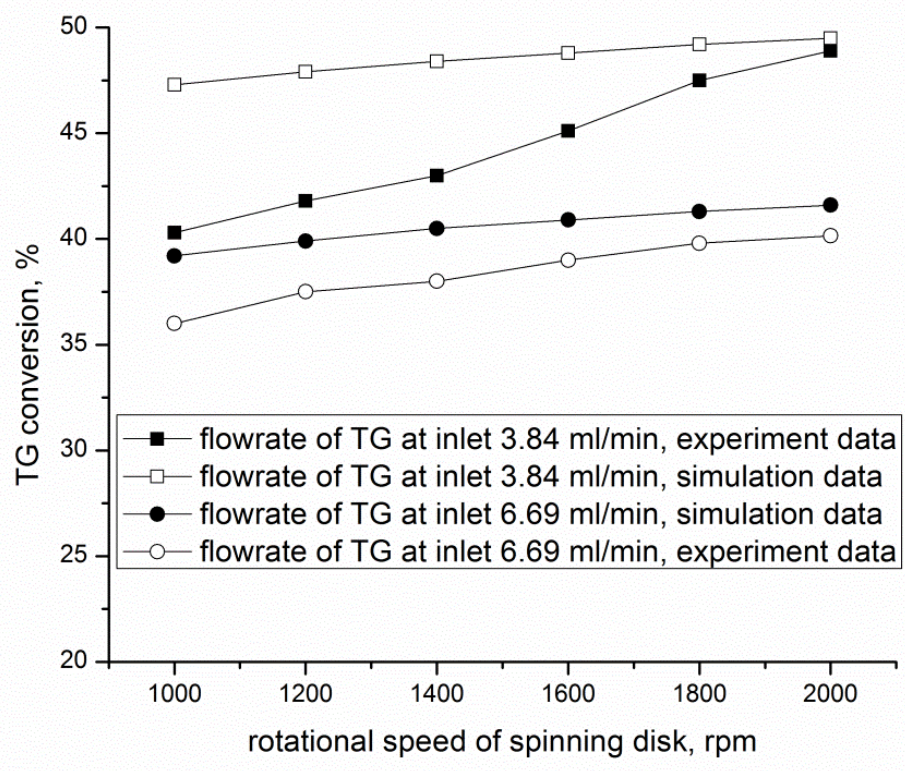

Fig. 13. Effect of TG flow rate at the inlet on TG conversion when gap size is $0.2 \mathrm{~mm}$

\section{CONCLUSIONS}

The CFD software ANSYSCFluent v.13.0 was successfully used to investigate the hydrodynamics inside SDR gap for biodiesel synthesis from canola oil and methanol in the presence of a sodium hydroxide catalyst. To accomplish this task, an adequate model was formulated and expressed in terms of facilities available within ANSYSCFluent. The simulating results were compared with corresponding experiment data obtained from a setup built by our collaborating partner from Kansas University. The modelling provides useful information on the reactor performance.

In the present research, liquids are injected into SDR and flow outwards at low radial positions, while at high radial positions there are two phenomena. The liquid accelerates near the rotor outwards because of centrifugal force caused by the spinning disk rotation, while a backflow near the stator is created as a result of the continuity conservation, which prevents the liquid from breakage.

In order to investigate effects of operating conditions on size of backflow zone, three gap sizes were simulated and for each gap size six rotational speeds of spinning disk were studied. For gap size $0.2 \mathrm{~mm}$, two flow rates of TG at inlet were simulated. The present study research well simulates the 
backflow phenomenon and explains why backflow is undesirable (resulting in lower TG conversion). Results show that the size of backflow zone increases as the rotational speed increases, and decreases with decreasing gap size between rotor and stator. Likewise size of backflow zone reduces with increasing flow rate of substrates at inlet.

The simulation also reveals that small gap size and fast rotational speed induce large shear force inside SDR, intensifying micro-mixing among reagents, consequently enhancing TG conversion. However, too fast rotational speed of the spinning disk leads to more backflow decreasing TG conversion, which is confirmed by experimental data. Hence, excessive rotational speeds are not recommended in practice.

What is more, large flow rate of TG at inlet is not recommended either. The mean residence time of the reactants in SDR is shorter than that when flow rate of TG at inlet is small. The mean residence time is too short for the reactants to react with large TG flow rate at inlet, consequently leads to smaller TG conversion.

Nevertheless spinning disk reactors are very effective devices intensifying the transesterification process for biodiesel synthesis. With the use of SDR, shorter process time and smaller equipment size is needed, which is very important for industrial production. We discussed the process time issue in our previous paper (Wen and Petera, 2015) confirming higher efficiency of SDR compared to the conventional apparatus. However, it is necessary to optimise the operating conditions in order to attain better biodiesel production efficiency as recommended above.

\section{SYMBOLS}

A correction factor

$A_{i} \quad$ pre-exponential factor, $\mathrm{m}^{3} \cdot \mathrm{kmol}^{-1} \cdot \mathrm{s}^{-1}$

a correction factor

$C_{0} \quad$ volume flow rate-average TG molar concentration at inlet, $\mathrm{kmol} \cdot \mathrm{m}^{-3}$

$C_{j, r} \quad$ molar concentration of species $j$ in reaction $r, \mathrm{kmol} \cdot \mathrm{m}^{-3}$

$C_{k} \quad$ volume flow rate-average TG molar concentration at outlet, $\mathrm{kmol} \cdot \mathrm{m}^{-3}$

$C_{\text {methanol }} \quad$ methanol concentration in the SDR gap, $\mathrm{kmol} \cdot \mathrm{m}^{-3}$

$C_{A i} \quad$ concentration of product at the interface, $\mathrm{kmol} \cdot \mathrm{m}^{-3}$

$C_{A} \quad$ concentration of product in the continuous phase, $\mathrm{kmol} \cdot \mathrm{m}^{-3}$

$C_{T G} \quad$ TG concentration in the SDR gap, $\mathrm{kmol} \cdot \mathrm{m}^{-3}$

$D \quad$ diameter of the reactor, $\mathrm{m}$

$D_{m, i 0} \quad$ pure diffusivities for individual components, $\mathrm{m}^{2} \mathrm{~s}^{-1}$

$D_{m, i} \quad$ effective diffusion coefficients for species $i$ in the mixture, $\mathrm{m}^{2} \mathrm{~s}^{-1}$

$d \quad$ gap size of the reactor, $\mathrm{m}$

$E_{i} \quad$ activation energy, $\mathrm{J} \cdot \mathrm{kmol}^{-1}$

$H_{i}^{0} \quad$ standard state enthalpy of species $i, \mathrm{~J} \cdot \mathrm{kmol}^{-1}$

$J_{i} \quad$ diffusion flux of species $i, \mathrm{~kg} \cdot \mathrm{m}^{-2} \cdot \mathrm{s}^{-1}$

$k_{b} \quad$ backward rate constant, $\mathrm{m}^{3} \cdot \mathrm{kmol}^{-1} \cdot \mathrm{s}^{-1}$

$k_{f} \quad$ forward rate constant, $\mathrm{m}^{3} \cdot \mathrm{kmol}^{-1} \cdot \mathrm{s}^{-1}$

$k_{b, r} \quad$ backward rate constant for the reaction $r, \mathrm{~m}^{3} \cdot \mathrm{kmol}^{-1} \cdot \mathrm{s}^{-1}$

$k_{f, r} \quad$ forward rate constant for the reaction $r, \mathrm{~m}^{3} \cdot \mathrm{kmol}^{-1} \cdot \mathrm{s}^{-1}$

$k_{i} \quad$ respective kinetic constant, $\mathrm{m}^{3} \cdot \mathrm{kmol}^{-1} \mathrm{~s}^{-1}$

$M_{w, i} \quad$ the molecular weight of species $i, \mathrm{~kg} \mathrm{kmol}^{-1}$

$N \quad$ rotational speed of spinning disk, rps

$N_{R} \quad$ total number of reactions that species $i$ participate in

$n \quad$ number of species 


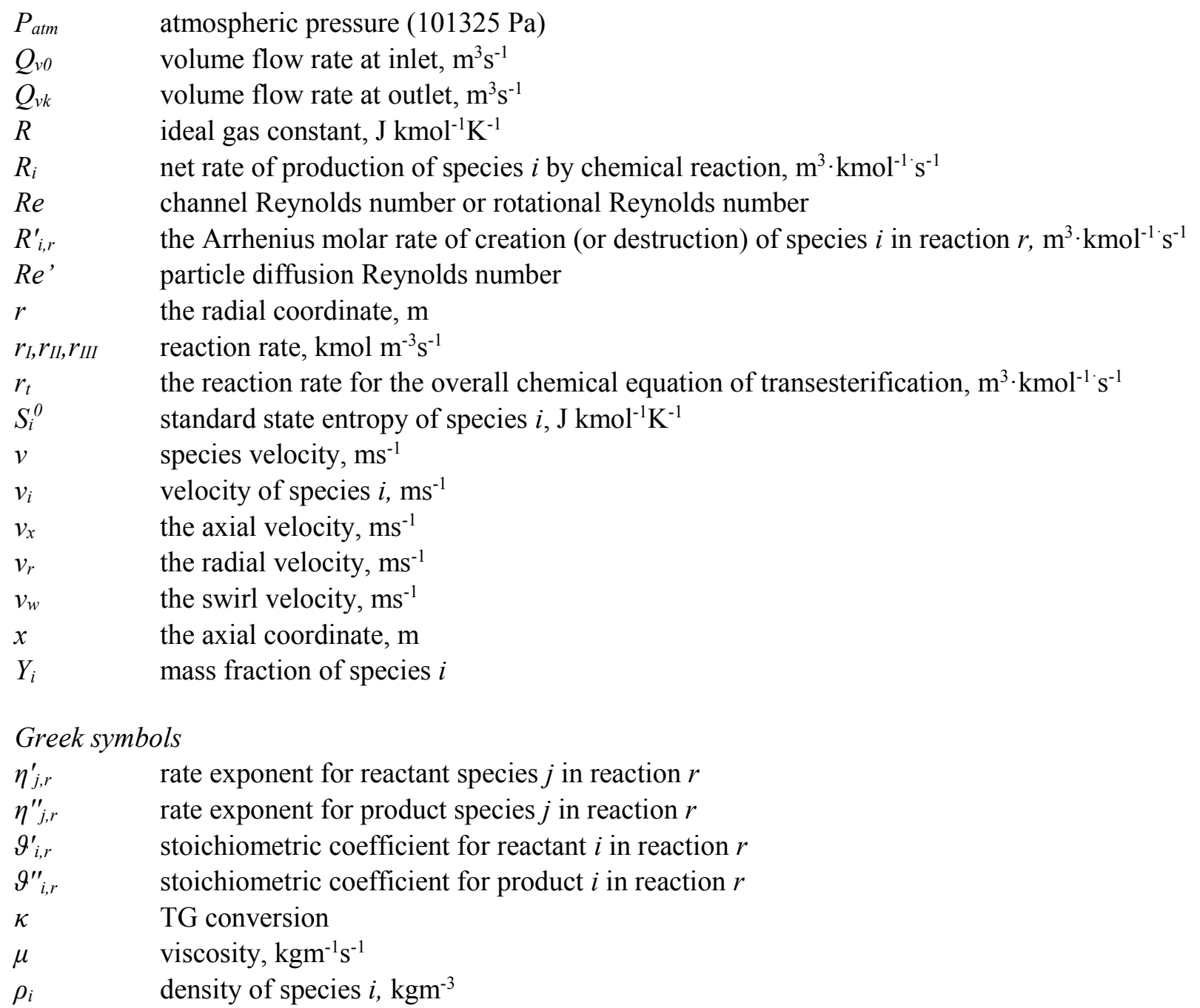

\section{REFERENCES}

Batchelor G., 1951. Note on a class of solutions of the Navier-Stokes equations representing steady rotationallysymmetric flow. Mech. Appl. Math., 4, 29-41. DOI: 10.1093/qjmam/4.1.29.

Bodewadt U.T., 1940. Die Drehströmung über dem festem Gründe. Z. Angew. Math. Mech., 20, 241-253.DOI: 10.1002/zamm.19400200502.

Brady J., Durlofsky L., 1987. On rotating disk flow. Fluid Mech., 175, 363-394. DOI: 10.1017/S0022112087000430.

Brechtelsbauer C., Lewis N., Oxley P., Ricard F., Ramshaw C., 2001. Evaluation of a spinning disc reactor for continuous processing. Org. Process Res. Dev., 5, 65-68. DOI: 10.1021/op0000834.

Cafiero L.M., Baffi G., Chianese A., Jachuck R.J., 2002. Process intensification: precipitation of barium sulphate using a spinning disc reactor. Ind. Eng. Chem. Res., 41, 5240-5246. DOI: 10.1021/ie010654w.

Clifford Y.T, Wang Y.H., Tai C.T., Liu H.S., 2009. Preparation of silver nanoparticles using a spinning disk reactor in a continuous mode. Ind. Eng. Chem. Res., 48, 10104-10109. DOI: 10.1021/ie9005645.

Cussler E.L., 1997. Diffusion: Mass transfer in fluid systems. 2nd edition. Cambridge University Press, NewYork, $111-120$

Daily J.W., Nece R.E., 1960. Chamber dimension effects on induced flow and frictional resistance of enclosed rotating disks. Basic Eng., 82, 217-232. DOI: 10.1115/1.3662532.

De Beer M.M., Keurentjes J.T.F., Schouten J.C., van der Schaaf J., 2014. Engineering model for single-phase flow in a multi-stage rotor-stator spinning disc reactor. Chem. Eng. J., 242, 53-61. DOI: 10.1016/j.cej.2013.12.052. 
Djaoui M., Dyment A., Debuchy R., 2001. Heat transfer in a rotor-stator system with a radial inflow. Eur. J. Mech. - B/Fluids, 20, 371-398. DOI: 10.1016/S0997-7546(01)01133-5.

Egbuna S.O, Ozonoh M, Aniokete T.C., 2013. Diffusion rate analysis in palm kernel oil extraction using differentextraction solvents. IJRET, 02, 639-648. DOI: 10.15623/ijret.2013.0211098.

Freedman B., Pryde E.H., Mounts T.L., 1984. Variables affecting the yields of fatty esters from transesterified vegetable oils. J. Am. Oil Chem. Soc., 61, 1638-1643. DOI: 10.1007/BF02541649.

Freedman B., Butterfield R.O., Pryde E.H., 1986. Transesterification kinetics of soybean oil. J. Am. Oil Chem. Soc., 63, 1375-1380. DOI: 10.1007/BF02679606.

Haddadi S., Poncet S., 2008. Turbulence modelling of torsional Couette flows. Int. J. Rotating Mach., 2008, 635138. DOI: $10.1155 / 2008 / 635138$.

Lopez J., 1998. Characteristics of endwall and sidewall boundary layers in a rotating cylinder with a differentially rotating endwall. Fluid Mech., 359, 49-79.DOI: 10.1017/S002211209700829X.

Noureddini H., Zhu D., 1997. Kinetics of transesterification of soybean oil. J. Am. Oil Chem. Soc., 74, 1457-1463. DOI: $10.1007 / \mathrm{s} 11746-997-0254-2$.

Phadke U., Owen J.M., 1988. Aerodynamic aspects of the sealing of gas-turbine rotor-stator systems. Part 1: The behavior of simple shrouded rotating-disk systems in a quiescent environment. Int. J. Heat Fluid Flow, 9 , 98-105. DOI: 10.1016/0142-727X(88)90060-4.

Poncet S., Chauve M.P., Le Gal P., 2005a. Turbulent rotating disk flow with inward throughflow. Fluid Mech., 522, 253-262. DOI: 10.1017/S0022112004002046.

Poncet S., Chauve M.P., Schiestel R., 2005b. Batchelor versus Stewartson flow structures in a rotor-stator cavity with throughflow. Phys. Fluids, 17, 075110. DOI: 10.1063/1.1964791.

Przybylski R., 2007. Canola oil: Physical and chemical properties. Canola council of Canada. Available at: http://www.canolacouncil.org/media/515239/canola_oil_physical_chemical_properties_1.pdf.

Qiu Z.Y., 2010. Intensification of liquid-liquid contacting processes. PhD Thesis, University of Kansas.

Qiu Z.Y., Petera J., Weatherley L.R., 2012. Biodiesel synthesis in an intensified spinning disc reactor. Chem. Eng.J., 210, 597-609. DOI: 10.1016/j.cej.2012.08.058.

Richardson, P. D., 1963. Heat and mass transfer in turbulent separated flows. Chem. Eng. Sci., 18: 149 -155.

Rudniak L., Machniewski P.M., Milewska A., Molga E., 2004. CFD modelling of stirred tank chemical reactors: homogeneous and heterogeneous reaction systems. Chem. Eng. Sci., 59, 5233-5239.

Rusconi R., Stone H.A., 2008. Shear-induced diffusion of platelike particles in microchannels. Phys. Rev. Lett., 101, 254502. DOI: 10.1103/PhysRevLett.101.254502.

Sandoval-Robles J.G., Riba J.P., Couderc J. P., 1980. Mass transfer around a sphere. Trans IChemE, 58, $132-134$. SAS IP, Inc., 2010. ANSYSCFluent v.13.0 Theory Guide.

Soo S.L., 1958. Laminar flow over an enclosed rotating disk. Trans. ASME, 80, 287-296.

Stewartson K., 1953. On the flow between two rotating coaxial disks. Math. Proc. Camb. Phil. Soc. 49, 333-341. DOI: $10.1017 / \mathrm{S} 0305004100028437$.

Von Kármán T., 1921. Über laminare und turbulente Reibung. Z. Angew. Math. Mech., 1(4), 233-252. DOI: 10.1002/zamm.19210010401.

Wen Z., Petera J., 2015. CFD Numerical simulation of biodiesel synthesis in a spinning disc reactor. Chem. Process Eng., 36, 21-37. DOI: 10.1515/cpe-2015-0002.

Zhou W., Bookcock D.G.B., 2006. Phase distributions of alcohol, glycerol, and catalyst in the transesterification of soybean oil. J. Am. Oil Chem. Soc., 83, 1047-1052. DOI: 10.1007/s11746-006-5161-4.

Received 01 December 2015

Received in revised form 02 February 2017

Accepted 19 February 2017 